\title{
Sum Relations for Multiple Zeta Values and Connection Formulas for the Gauss Hypergeometric Functions
}

\author{
By \\ Takashi AokI * and Yasuo OHNO**
}

\begin{abstract}
We give an explicit representation for the sums of multiple zeta-star values of fixed weight and height in terms of Riemann zeta values.
\end{abstract}

\section{$\S 1 . \quad$ Introduction}

In this article we establish a new family of relations between sums of multiple zeta values and Riemann zeta values. This family contains relations which do not appear in the families of relations given in [6], [9], [11].

Concerning multiple zeta values, there are two types of definition: multiple zeta values defined by the Euler sums with strict inequalities in the summation and defined by those with non-strict inequalities (see below). The former is mainly used in mathematical literature and the latter is the main subject of this article. Normally multiple zeta values ( $M Z V s$ for short) mean the former and are denoted by $\zeta(\mathbf{k})$. We tentatively call the latter multiple zeta-star values and denote them by $\zeta^{*}(\mathbf{k})$ to distinguish them from ordinary ones. We abbreviate them to MZSVs. They are classic objects although there had been no name of them. In fact, Euler was the first mathematician who was interested in multiple zeta values and he mainly treated MZSVs [3]. Recently, Hoffman [5] pointed

Communicated by T. Kawai. Received September 19, 2003. Revised January 19, 2004. 2000 Mathematics Subject Classification(s): 11M06, $40 \mathrm{~B} 05$.

Department of Mathematics, Kinki University, Higashi-Osaka, Osaka 577-8502, Japan.

*Supported in part by JSPS Grant-in-Aid No. 14340042 and by No. 15540190

e-mail: aoki@math.kindai.ac.jp

**Supported in part by JSPS Grant-in-Aid No. 15740025 and by No. 15540190

e-mail: ohno@math.kindai.ac.jp

(C) 2005 Research Institute for Mathematical Sciences, Kyoto University. All rights reserved. 
out the significance of considering MZSVs as well as MZVs. The notation $S$ was used there instead of $\zeta^{*}$.

The main result of this article shows that the sum of MZSVs with fixed weight and height turns out to be a rational multiple of the Riemann zeta value of the same weight. Considering MZSVs clarifies the importance of those two indices: weight and height. They have been played a role in [8], [11]. The employment of the indices and MZSVs is a neat way to formulate systematic description of relations that hold among MZVs. Another important index is depth. We believe that MZSVs and the three indices: weight, height and depth will play an important role in investigation of the structure of $\mathbf{Q}$-algebra generated by MZVs. (Note that this algebra coincides with $\mathbf{Q}$-algebra generated by MZSVs.)

An interesting feature of the method employed in our proof is related to the theory of differential equations in the complex domain. The method is a variation on [11] and the use of connection formulas of the Gauss hypergeometric function is essential in both cases (see [7], [12] also).

\section{$\S 2 . \quad$ Statement of the Results}

For any multi-index $\mathbf{k}=\left(k_{1}, k_{2}, \ldots, k_{n}\right)\left(k_{i} \in \mathbf{Z}, k_{i}>0\right)$, the weight, depth, and height of $\mathbf{k}$ are by definition the integers $k=k_{1}+k_{2}+\cdots+k_{n}, n$, and $s=\#\left\{i \mid k_{i}>1\right\}$, respectively. We denote by $I(k, s)$ the set of multi-indices $\mathbf{k}$ of weight $k$ and height $s$, and by $I_{0}(k, s)$ the subset of admissible indices, i.e., indices with the extra requirement that $k_{1} \geq 2$. For any admissible index $\mathbf{k}=\left(k_{1}, k_{2}, \ldots, k_{n}\right) \in I_{0}(k, s)$, the multiple zeta values $\zeta^{*}(\mathbf{k})$ and $\zeta(\mathbf{k})$ are defined by

$$
\begin{aligned}
& \zeta^{*}(\mathbf{k})=\zeta^{*}\left(k_{1}, k_{2}, \ldots, k_{n}\right)=\sum_{m_{1} \geq m_{2} \geq \cdots \geq m_{n} \geq 1} \frac{1}{m_{1} k_{1} m_{2} k_{2} \cdots m_{n} k_{n}}, \\
& \zeta(\mathbf{k})=\zeta\left(k_{1}, k_{2}, \ldots, k_{n}\right)=\sum_{m_{1}>m_{2}>\cdots>m_{n}>0} \frac{1}{m_{1} k_{1} m_{2} k_{2} \cdots m_{n} k_{n}} .
\end{aligned}
$$

Note that, there are linear relations among $\zeta^{*}$ and $\zeta$, for example,

$$
\begin{aligned}
\zeta^{*}\left(k_{1}, k_{2}\right)= & \zeta\left(k_{1}, k_{2}\right)+\zeta\left(k_{1}+k_{2}\right), \quad \zeta\left(k_{1}, k_{2}\right)=\zeta^{*}\left(k_{1}, k_{2}\right)-\zeta^{*}\left(k_{1}+k_{2}\right), \\
\zeta^{*}\left(k_{1}, k_{2}, k_{3}\right)= & \zeta\left(k_{1}, k_{2}, k_{3}\right)+\zeta\left(k_{1}+k_{2}, k_{3}\right)+\zeta\left(k_{1}, k_{2}+k_{3}\right)+\zeta\left(k_{1}+k_{2}+k_{3}\right), \\
\zeta\left(k_{1}, k_{2}, k_{3}\right)= & \zeta^{*}\left(k_{1}, k_{2}, k_{3}\right)-\zeta^{*}\left(k_{1}+k_{2}, k_{3}\right) \\
& -\zeta^{*}\left(k_{1}, k_{2}+k_{3}\right)+\zeta^{*}\left(k_{1}+k_{2}+k_{3}\right),
\end{aligned}
$$


and so on. Multiple zeta-star values $\zeta^{*}(\mathbf{k})$ had been studied by Euler [3], and his study is the origin of various researches of multiple zeta values. We consider the sum of multiple zeta-star values of fixed weight and height:

$$
\sum_{\mathbf{k} \in I_{0}(k, s)} \zeta^{*}(\mathbf{k})
$$

Our main result will then be

Theorem 1. The sum (1) is given by

$$
\sum_{\mathbf{k} \in I_{0}(k, s)} \zeta^{*}(\mathbf{k})=2\left(\begin{array}{c}
k-1 \\
2 s-1
\end{array}\right)\left(1-2^{1-k}\right) \zeta(k) .
$$

Remark 1. If we replace $\zeta^{*}$ by $\zeta$ in the left-hand side of (2), it is unlikely that the value is expressed as a rational multiple of $\zeta(k)$. For example, in case when $k=5$ and $s=1$, the left-hand side becomes

$$
\zeta(5)+\zeta(4,1)+\zeta(3,1,1)+\zeta(2,1,1,1)=6 \zeta(5)-2 \zeta(2) \zeta(3) .
$$

It has been proved by Le and Murakami [8] that the following relation

$$
\sum_{\mathbf{k} \in I_{0}(2 k, s)}(-1)^{\operatorname{dep}(\mathbf{k})} \zeta(\mathbf{k})=\frac{(-1)^{k}}{(2 k+1) !} \sum_{r=0}^{k-s}\left(\begin{array}{c}
2 k+1 \\
2 r
\end{array}\right)\left(2-2^{2 r}\right) B_{2 r} \pi^{2 k}
$$

holds for a fixed even weight and a fixed height, where $B_{n}$ denotes the $n$-th Bernoulli number and $\operatorname{dep}(\mathbf{k})$ denotes the depth of $\mathbf{k}$. If we rewrite the lefthand side of $(3)$ in terms of $\zeta^{*}$, the height of each term is not equal any more. Therefore the left-hand side after the replacement does not take the same form as the left-hand side of (2). Note that (3) is a special case of the result given in [11], where a generating function for sums of MZVs with fixed weight, depth and height is constructed.

As an application of Theorem 1, we can express sums of special values of Arakawa-Kaneko zeta function in terms of Riemann zeta values.

For any positive integer $k \geq 1$, T. Arakawa and M. Kaneko [1] defined the function $\xi_{k}(s)$ by

$$
\xi_{k}(s)=\frac{1}{\Gamma(s)} \int_{0}^{\infty} \frac{t^{s-1}}{e^{t}-1} \operatorname{Li}_{k}\left(1-e^{-t}\right) d t,
$$

where $\operatorname{Li}_{k}(s)$ denotes the $k$-th polylogarithm $\operatorname{Li}_{k}(s)=\sum_{m=1}^{\infty} \frac{s^{m}}{m^{k}}$. The integral converges for $\operatorname{Re}(s)>0$ and the function $\xi_{k}(s)$ continues to an entire function 
of whole $s$-plane. They proved that the special values of $\xi_{k}(s)$ at non-positive integers are given by poly-Bernoulli numbers and the values at positive integers are given in terms of multiple zeta values. Thereafter the second author [9] gave the following relation among the values of $\xi_{k}(s)$ at positive integers and MZSVs:

$$
\xi_{k}(n)=\zeta^{*}(k+1, \underbrace{1, \ldots, 1}_{n-1})
$$

where the both indices $k$ and $n$ are positive integers. By using this relation, we have the following corollary of Theorem 1.

Corollary 1. For any integer $k>1$, we have

$$
\sum_{n=1}^{k-1} \xi_{k-n}(n)=2(k-1)\left(1-2^{1-k}\right) \zeta(k)
$$

Or equivalently,

Corollary 2. For any integer $k>1$, we have

$$
\zeta(k)=\frac{1}{2(k-1)\left(1-2^{1-k}\right)} \int_{0}^{\infty} \frac{1}{e^{t}-1} \sum_{n=1}^{k-1} \frac{t^{n-1}}{(n-1) !} \operatorname{Li}_{k-n}\left(1-e^{-t}\right) d t .
$$

\section{§3. Proof of Theorem 1}

We denote by $X_{0}(k, s)$ the left-hand side of (2):

$$
X_{0}(k, s)=\sum_{\mathbf{k} \in I_{0}(k, s)} \zeta^{*}(\mathbf{k}) .
$$

Since the set $I_{0}(k, s)$ is non-empty only if the indices $k$ and $s$ satisfy the inequalities $s \geq 1$ and $k \geq 2 s$, we can collect all the numbers $X_{0}(k, s)$ into a single generating function

$$
\Phi_{0}(x, z)=\sum_{k, s} X_{0}(k, s) x^{k-2 s} z^{2 s-2} \in \mathbf{R}[[x, z]] .
$$

Following [11] and [14], we consider the multiple zeta-star value $\zeta^{*}(\mathbf{k})$ as the limiting value at $t=1$ of the function

$$
L_{\mathbf{k}}^{*}(t)=L_{k_{1}, k_{2}, \ldots, k_{n}}^{*}(t)=\sum_{m_{1} \geq m_{2} \geq \cdots \geq m_{n} \geq 1} \frac{t^{m_{1}}}{m_{1}^{k_{1}} m_{2}^{k_{2}} \cdots m_{n} k_{n}} \quad(|t|<1) .
$$


Note that we consider $L_{\mathbf{k}}^{*}(t)$ not just for $\mathbf{k} \in I_{0}$ but for all $\mathbf{k} \in I$. For $\mathbf{k}$ empty we define $L_{\mathbf{k}}^{*}(t)$ to be 1 . For non-negative integers $k$ and $s$ set

$$
X(k, s ; t)=\sum_{\mathbf{k} \in I(k, s)} L_{\mathbf{k}}^{*}(t)
$$

(so $X(0,0 ; t)=1$ and $X(k, s ; t)=0$ unless $k \geq 2 s$ and $s \geq 0$ ), and let $X_{0}(k, s ; t)$ be the function defined by the same formula but with the summation restricted to $\mathbf{k} \in I_{0}(k, s)$.

We denote by $\Phi=\Phi(x, z ; t)$ and $\Phi_{0}=\Phi_{0}(x, z ; t)$ the corresponding generating functions

$$
\Phi=\sum_{k, s \geq 0} X(k, s ; t) x^{k-2 s} z^{2 s}=1+L_{1}^{*}(t) x+L_{1,1}^{*}(t) x^{2}+\cdots
$$

and

$$
\Phi_{0}=\sum_{k, s \geq 0} X_{0}(k, s ; t) x^{k-2 s} z^{2 s-2}=L_{2}^{*}(t)+L_{2,1}^{*}(t) x+L_{3}^{*}(t) x+\cdots
$$

Note that the coefficient of $x^{k-2 s} z^{2 s-2}$ in $\Phi_{0}(x, z ; 1)=\Phi_{0}(x, z)$ is $X_{0}(k, s)$. Using the formulas

$$
\frac{d}{d t} L_{k_{1}, \ldots, k_{n}}^{*}(t)= \begin{cases}\frac{1}{t} L_{k_{1}-1, k_{2}, \ldots, k_{n}}^{*}(t) & \text { if } \quad k_{1} \geq 2, \\ \frac{1}{t(1-t)} L_{k_{2}, k_{3}, \ldots, k_{n}}^{*}(t) & \text { if } \quad k_{1}=1, n>1\end{cases}
$$

and

$$
\frac{d}{d t} L_{1}^{*}(t)=\frac{1}{1-t}
$$

for the derivative of $L_{\mathbf{k}}^{*}(t)$, we obtain

$$
\begin{aligned}
\frac{d}{d t} X_{0}(k, s ; t)= & \frac{1}{t}\left(X(k-1, s-1 ; t)-X_{0}(k-1, s-1 ; t)+X_{0}(k-1, s ; t)\right), \\
& \frac{d}{d t}\left(X(k, s ; t)-X_{0}(k, s ; t)\right)=\frac{1}{t(1-t)} X(k-1, s ; t)
\end{aligned}
$$

or, in terms of generating functions,

$$
\frac{d \Phi_{0}}{d t}=\frac{1}{x t}\left(\Phi-1-z^{2} \Phi_{0}\right)+\frac{x}{t} \Phi_{0}, \quad \frac{d}{d t}\left(\Phi-z^{2} \Phi_{0}\right)=\frac{x}{t(1-t)}(\Phi-1)+\frac{x}{1-t} .
$$


Eliminating $\Phi$, we obtain the differential equation

$$
t^{2}(1-t) \frac{d^{2} \Phi_{0}}{d t^{2}}+t((1-t)(1-x)-x) \frac{d \Phi_{0}}{d t}+\left(x^{2}-z^{2}\right) \Phi_{0}=t
$$

for the power series $\Phi_{0}$. The unique power-series solution at $t=0$ is given by

$$
\Phi_{0}(x, z ; t)=\sum_{n=1}^{\infty} a_{n} t^{n}
$$

with

$$
a_{n}=\frac{\Gamma(n) \Gamma(n-x) \Gamma(1-x-z) \Gamma(1-x+z)}{\Gamma(1-x) \Gamma(1-x-z+n) \Gamma(1-x+z+n)} .
$$

Here $\Gamma(z)$ denotes the gamma function. Specializing to $t=1$ gives

$$
\Phi_{0}(x, z ; 1)=\sum_{n=1}^{\infty} a_{n}
$$

We need to evaluate the right-hand side of (7). We can rewrite $a_{n}$ in the form

$$
a_{n}=\sum_{l=1}^{n}\left(\frac{A_{n, l}^{(+)}}{x+z-l}+\frac{A_{n, l}^{(-)}}{x-z-l}\right)
$$

with

$$
A_{n, l}^{( \pm)}=(-1)^{l}\left(\begin{array}{c}
n-1 \\
l-1
\end{array}\right) \frac{( \pm z-l+1)( \pm z-l+2) \cdots( \pm z-l+n-1)}{( \pm 2 z-l+1)( \pm 2 z-l+2) \cdots( \pm 2 z-l+n)} .
$$

Hence we have

$$
\begin{aligned}
\sum_{n=1}^{\infty} a_{n} & =\sum_{n=1}^{\infty} \sum_{l=1}^{n}\left(\frac{A_{n, l}^{(+)}}{x+z-l}+\frac{A_{n, l}^{(-)}}{x-z-l}\right) \\
& =\sum_{l=1}^{n}\left(\sum_{n=l}^{\infty} A_{n, l}^{(+)} \frac{1}{x+z-l}+\sum_{n=l}^{\infty} A_{n, l}^{(-)} \frac{1}{x-z-l}\right) .
\end{aligned}
$$

The sums of $A_{n, l}^{( \pm)}$in $n$ are evaluated as follows:

$$
\begin{aligned}
\sum_{n=l}^{\infty} A_{n, l}^{( \pm)} & =(-1)^{l} \sum_{n=0}^{\infty} \frac{(l-1+n) !( \pm z-l+1)( \pm z-l+2) \cdots( \pm z+n-1)}{n !(l-1) !( \pm 2 z-l+1)( \pm 2 z-l+2) \cdots( \pm 2 z+n)} \\
& =(-1)^{l} \frac{( \pm z-l+1)( \pm z-l+2) \cdots( \pm z-1)}{( \pm 2 z-l+1)( \pm 2 z-l+2) \cdots( \pm 2 z)} F(l, \pm z, \pm 2 z+1,1)
\end{aligned}
$$


where $F(\alpha, \beta, \gamma ; t)$ denotes the Gauss hypergeometric function. Using Gauss' formula for $F(\alpha, \beta, \gamma ; 1)$ gives

$$
\sum_{n=l}^{\infty} A_{n, l}^{( \pm)}= \pm \frac{(-1)^{l}}{z}
$$

Hence we have

$$
\sum_{n=1}^{\infty} a_{n}=\frac{1}{z} \sum_{l=1}^{\infty}(-1)^{l}\left(\frac{1}{x+z-l}-\frac{1}{x-z-l}\right) .
$$

Expanding the right-hand side in power series of $x$ and $z$ and taking the coefficient of $x^{k-2 s} z^{2 s-2}(\mathrm{cf}$. (5)) gives

$$
2\left(\begin{array}{c}
k-1 \\
2 s-1
\end{array}\right) \sum_{l=1}^{\infty} \frac{(-1)^{l-1}}{l^{k}}
$$

and now using the relation $\sum_{l=1}^{\infty} \frac{(-1)^{l-1}}{l^{k}}=\left(1-2^{1-k}\right) \zeta(k)$ yields equation $(2)$.

\section{Appendix}

The relation given in Theorem 1 can be interpreted as an equality concerning an integral which contains the Gauss hypergeometric function.

Theorem 2. Under suitable conditions for the parameters $x$ and $z$ that guarantee existence of both members, the following equality holds:

$$
\begin{aligned}
& \frac{1}{1-x} \int_{0}^{1}(1-t)^{z-x} F(1-x+z, 1+z, 2-x ; t) d t \\
&=\frac{1}{z} \sum_{l=1}^{\infty}(-1)^{l}\left(\frac{1}{x+z-l}-\frac{1}{x-z-l}\right) .
\end{aligned}
$$

Proof. We set

$$
\begin{aligned}
& \phi_{1}(t)=t^{x+z} F(x+z, z, 2 z+1 ; t), \\
& \phi_{2}(t)=t^{x-z} F(x-z,-z,-2 z+1 ; t) .
\end{aligned}
$$

Then $\left(\phi_{1}, \phi_{2}\right)$ is a system of fundamental solutions of the homogeneous equation of (6). The unique holomorphic solution $\Phi_{0}$ of (6) is constructed in the form

$$
\Phi_{0}=u_{1} \phi_{1}+u_{2} \phi_{2}
$$


where $u_{1}$ and $u_{2}$ are defined as follows:

$$
\begin{aligned}
& u_{1}(t)=\frac{1}{2 z} \int_{0}^{t} s^{-x-z}(1-s)^{x-1} F(x-z,-z,-2 z+1 ; s) d s, \\
& u_{2}(t)=-\frac{1}{2 z} \int_{0}^{t} s^{-x+z}(1-s)^{x-1} F(x+z, z, 2 z+1 ; s) d s .
\end{aligned}
$$

The values $\phi_{1}(1)$ and $\phi_{2}(1)$ are obtained by using Gauss' formula. Hence we find that $\Phi_{0}(1)=u_{1}(1) \phi_{1}(1)+u_{2}(1) \phi_{2}(1)$ has the value

$$
\begin{array}{r}
\int_{0}^{1} d t(1-t)^{x-1} t^{-x+z}\left\{\frac{\Gamma(-2 z) \Gamma(1-x)}{\Gamma(1-x-z) \Gamma(1-z)} F(x+z, z, 2 z+1 ; t)\right. \\
\left.+\frac{\Gamma(2 z) \Gamma(1-x)}{\Gamma(1-x+z) \Gamma(1+z)} t^{z-x} F(x-z,-z,-2 z+1 ; t)\right\} .
\end{array}
$$

Using one of the connection formulas for the Gauss hypergeometric functions (e.g., (43), p. 108 in [2]) yields

$$
\Phi_{0}(1)=\frac{1}{1-x} \int_{0}^{1} t^{z-x} F(1-x+z, 1+z, 2-x ; 1-t) d t,
$$

which is equal to the left-hand side of (8). The right-hand side has been already obtained in the proof of Theorem 1 . This proves Theorem 2.

Remark 2. The right-hand side of (8) can be written in the following form:

$$
-\frac{1}{z}\left(\psi(1-(x+z))-\psi(1-(x-z))-\psi\left(1-\frac{x+z}{2}\right)+\psi\left(1-\frac{x-z}{2}\right)\right),
$$

where $\psi(t)=\frac{\Gamma^{\prime}(t)}{\Gamma(t)}$ is the di-gamma function.

\section{References}

[1] Arakawa, T. and Kaneko, M., Multiple zeta-values, poly-Bernoulli numbers, and related zeta functions, Nagoya Math. J., 153 (1999), 1-21.

[2] Erdérly, A., (eds.), Higher transcendental functions, vol. 1, Robert E. Krieger Publishing Company, Malabar, 1985.

[3] Euler, L., Meditationes circa singulare serierum genus, Novi Comm. Acad. Sci. Petropol 20 (1775), 140-186, reprinted in Opera Omnia ser. I, vol. 15, B. G. Teubner, Berlin (1927), 217-267.

[4] Granville, A., A decomposition of Riemann's Zeta-Function, in Analytic Number Theory, London Math. Soc. Lecture Note Ser., 247, Y. Motohashi (ed.), Cambridge University Press, (1997), 95-101. 
[5] Hoffman, M., Multiple Harmonic series, Pacific J. Math., 152 (1992), 275-290.

[6] Hoffman, M. and Ohno, Y., Relations of multiple zeta values and their algebraic expression, J. Algebra, 262 (2003), 332-347.

[7] Kombu, Y., Multiple zeta values and hypergeometric differential equations (in Japanese), Kinki University master's thesis (2003).

[8] Le, T. Q. T. and Murakami, J., Kontsevich's integral for the Homfly polynomial and relations between values of multiple zeta functions, Topology Appl., 62 (1995), 193-206.

[9] Ohno, Y., A generalization of the duality and sum formulas on the multiple zeta values, J. Number Theory, 74 (1999), 39-43.

[10] Sum formulas on multiple zeta values, in Zeta Functions, Topology and Quantum Physics, Dev. Math., 14 (2005), 131-144.

[11] Ohno, Y. and Zagier, D., Multiple zeta values of fixed weight, depth, and height, Indag. Math., 12 (2001), 483-487.

[12] J. Okuda and K. Ueno, Relations for multiple zeta values and Mellin transforms of multiple polylogarithms, Publ. RIMS, Kyoto Univ., 40 (2004), 537-564.

[13] Zagier, D., Values of zeta functions and their applications, in Proceedings of ECM 1992, Progr. Math., 120 (1994), 497-512.

[14] Multiple zeta values, unpublished preprint, Bonn, 1995. 